\title{
Authors' reply to commentators
}

We are grateful to the editors of SPM for having invited comments on our report and to the distinguished commentators for their thoughtful remarks. We appreciate the opportunity that is given us here to respond.

The remarks of van den Brink, Davoli and Perucci, Verthein et al. and Monteiro are mostly complimentary of, and largely complementary to, our report and, in that sense, do not require extensive replies. The criticisms of Newman will be dealt with in more detail.

Van den Brink's comments are generally supportive of our approach and conclusions, though he does regret certain absences in the paper. It is correct that stage of illness (as opposed to stage of treatment) and treatment objectives are not explicitly included, as such. This is because we felt that the modular approach in our scenarios allows the therapists to implicitly consider illness severity and the therapeutic response to targeted problems.

Van den Brink has correctly made the conceptual distinction between cure and care. However, it is important to emphasise that in real life, even if cure is the eventual goal, care (i.e., MMT) may be used for different reasons on the difficult pathway leading to abstinence.

Physical examination and blood tests are not listed in our list of initial assessments, though they could very well have been. The reason, as van den Brink understands, is because in our system the first level of treatment is with the primary care physician and it is assumed that this assessment has been done if the patient's condition and physician's need for information requires. Physical examination and blood tests were thus considered to be part of usual care and left out of the framework of the specific criteria examined. As can be seen from the actual ratings, all the elements we identified for initial assessment were considered appropriate by the expert panel. A further element that van den Brink misses is substitution with other opioids. There are at least two reasons for this

1. more than $90 \%$ of maintenance/substitution is based on methadone, and
2. the other substances are more generally used in case of failure of methadone.

Concerning comparable figures of disagreement, we can confirm that the figures for disagreement (and uncertainty) are, in fact, rather low compared to previous experience (ours and others) in applying the RAND method to healthcare interventions. For example, panels that some of us (IPV, $B B$ ) have performed have shown disagreement figures of $6 \%$ for laminectomy (Vader et al. 2000), 14\% for colonoscopy (Burnand et al. 1998), $8 \%$ for gastroscopy (Vader et al. 1997).

Monteiro's perspective from the World Health Organization is enlightening and we have little to add to his remarks. We can only echo his insistence that "research continues to be a priority" in this field and his reminder that such research must be at once well-designed, well-conducted and close to the reality of every-day practice. We are also acutely aware, as Monteiro reminds us, that the difficulties encountered in changing clinical practice are a major obstacle to improving the quality of care and that this will require a wide range of incentives in various components and at all levels of our health care systems.

The call for international collaboration is vital and is reflected in our own recommendation to the Swiss authorities that they foster and intensify such collaboration. It is hoped that the insights provided from this experience in one healthcare system may indeed "serve as an incentive to donors, international organisations and research institutions to mobilize resources needed to improve the care of people with heroin dependence."

Verthein et al. have underlined important areas where there were higher levels of disagreement in the Swiss expert panel and have linked that disagreement to the fact that these are precisely areas that are begging for high-quality research. Such areas include "psycho-social interventions", "co-treatment" and the major question of "treatment withdrawal". 
Concerning regulation, we understand the sensitive nature of regulation in an area that is highly charged emotionally and the delicate balance required in providing regulation that is as little as possible, but as much as necessary. The political context here cannot be ignored and we do hope that the bringing together of experts with differing perspectives, differing fields of expertise and differing approaches will act as a catalyst for the wider arena to find consensus where feasible and to initiate and fund the necessary research to answer the questions where consensus is not yet possible.

As Verthein et al. point out, the Swiss context is exemplary in some respects. We have enjoyed a relative (to other nations) liberal attitude towards prescription and financing of MMT, especially in the framework of combating the AIDS/HIV epidemic. Among the encouraging results is "coverage" of substitution among the opiate-dependent population which is among the highest in the world, attaining about ${ }^{2}{ }_{3}$ of the opiate-dependents.

Davoli and Perucci, from their unique perspective of the Cochrane Collaboration, give us a healthy reminder of the many questions which are not answered in our report and most of which still beg for clear answers from research. They underline the important point that the indication for MMT must take place within a network of care and requires both medical and social consensus.

Concerning the important distinction between evidence-based recommendations and recommendations based on clinical or subjective judgement, or, as in our case, from panel judgement, we concur that this is important. For that reason, we strongly recommend that the results from the panel be read, as they were developed, i.e., in conjunction with the Swiss and international literature reviews which are freely available on the internet. We feel moved to add, however, that an ideal world where every question in health care will have a timely answer based on methodologically sound research will probably never exist. We therefore need methods to support or guide health care decisions that will, of necessity, be based on lower quality evidence.

The commentary from Newman is the only one that is clearly critical of the report. For this reason, we will allow ourselves to dwell in more detail on his remarks. Admittedly, he had difficulties understanding the objectives and the method and we feel that several of his remarks are due to misunderstandings.

Newman's finds incomprehensible our use of the concept of "post-cure phase of treatment". We assumed it was obvious that we were not using "cure" in the sense he implies ("addiction is incurable"), but rather in the alternative meaning as "a course or period of treatment". In that sense, the "postcure" phase of care would be that period following the with- drawal of methadone maintenance treatment, if and when the patient and/or therapist decides that it should be withdrawn. This phase obviously requires special attention. Newman also takes issue with our recommendation that nationally accepted practice guidelines be developed, "disseminated, promoted and used, and rigorously evaluated for compliance and outcome." He questions the necessity of such a process, given the existence of national guidelines in 17 countries.

There are many reasons why the process is necessary. Some of the problems with current guidelines are identified in the commentaries by van den Brink, Monteiro, Davoli and Perucci. Simply because 17 countries (including Switzerland) have previously developed practice guidelines for methadone maintenance treatment is by no means a guarantee that those guidelines are up-to-date, scientifically sound, concordant in their detailed recommendations, relevant to the Swiss context, complete in their coverage of the important questions or acceptable to those who are called upon to use them. The science of guidelines development and evaluation is really in its infancy and, given the on-going evolution of knowledge, there will always be a need to renew, update or adapt guidelines.

As to the need for "rigorous evaluation" of compliance and outcome, Newman seems to have misunderstood this to mean "rigorous enforcement" of compliance. That is not what we wrote, nor what we intended. We hope that, with so many open questions in this vital field of health care, Newman will have no trouble understanding our call for "rigorous evaluation" of both compliance and outcome. As the international and national literature reviews (referenced in our paper and freely available on the web) indicated, this field is plagued with an over-abundance of poor quality studies and a paucity of high-quality research. No evaluation of the "outcome" of guidelines implementation has any sense if there is not a simultaneous - and rigorous - evaluation of the "compliance" with those guidelines. Otherwise, there is no possible way to establish the link between the desired outcome (or its absence) and the guidelines themselves. For example, one set of patients may fair poorly because the guidelines were simply bad (and should be discarded) or because the guidelines were good but were not followed. The rigorous evaluation required to answer that fundamental question has been absent in so much work on the quality of practice guidelines in healthcare.

Newman finds that the conclusions of the report range from the self-evident to the incomprehensible, and he questions the qualifications of the experts identified for the panel. What may be self-evident to a highly accomplished international expert like Newman, may not be for the primary care

Soz.- Präventivmed. 48 Suppl 1(2003) S25-S27

(c) Birkhäuser Verlag, Basel, 2003 
physician in the field who will be prescribing methadone and caring for the dependent patient.

A word about the Swiss context may also help clear up misunderstandings. We deliberately chose a panel of Swiss experts, from a variety of backgrounds, rather than a panel of internationally renowned academics, because we were concerned about the acceptance of guidelines in the Swiss context. In Switzerland, methadone prescription is not reserved for specialists, nor decided in closed scientific circles. Rather, it is undertaken in therapeutic networks with the primary care physician at the centre. This requires consensus and cooperation, on the front lines, with social workers, pharmacists, psychologists and others. Without such collaboration and consensus, treatment is foredoomed to failure. It is because of this approach that the coverage of substitution treatment has attained a relatively high level in our country. It is also because of that context that the "experts" we identified were not a group of ivory-tower academics - though some are accomplished academics -, but a representative group of respected therapists, responsible for the prescription of methadone in a multidisciplinary network.

This is also in keeping with the RAND panel approach which seeks to combine scientific evidence (found in the literature review) with clinical expertise (present in our panel). We hope the above explanations will assist Newman, and others who may have similar misgivings, to better understand the work that was done and the reasons for the approach taken. We thank Newman for his remarks which allowed us to address these points.

\author{
References \\ Burnand B, Vader JP, Froehlich F, et al. (1998). \\ Reliability of panel based guidelines for colonos- \\ copy: an international comparison. Gastrointest \\ Endosc 47: 162-6.
}

Vader JP, Burnand B, Froehlich F, et al. (1997). Appropriateness of upper gastrointestinal endoscopy: comparison of American and Swiss criteria. Int J Qual Health Care 9: 87-92.
Vader JP, Porchet F, Larequi-Lauber T, Dubois $R W$, Burnand B (2000). Appropriateness of surgery for sciatica: reliability of guidelines from expert panels. Spine 25: 1831-6.

Soz.- Präventivmed. 48 Supple 1 (2003) S25-S27

(c) Birkhäuser Verlag, Basel, 2003 